\title{
Quarkonium production in pp and p-A collisions with ALICE at the LHC
}

\author{
Astrid Morreale for the ALICE collaboration ${ }^{1, \star}$ \\ ${ }^{1}$ Laboratoire de Physique Subatomique et des Technologies Associees SUBATECH. 4 Rue Alfred Kastler, \\ 44300 Nantes, France
}

\begin{abstract}
Quarkonia are mesons formed of either a charm and anti-charm quark pair $(\mathrm{J} / \psi, \psi(2 S))$ or a beauty and anti-beauty quark pair ( $(1 \mathrm{~S}),(2 \mathrm{~S})$ and $(3 \mathrm{~S}))$. We report on forward rapidity $(2.5<y<4) \mathrm{J} / \psi$ and $\psi(2 S)$ production measured in pp collisions at $\sqrt{s}=13 \mathrm{TeV}$, using data collected at the LHC in 2015. The results will be compared with similar measurements performed at $\sqrt{s}=2.76,5.02,7$ and $8 \mathrm{TeV}$. They will be further compared to NRQCD and FONLL calculations, which describe prompt and non-prompt charmonium production respectively. Results of the $\mathrm{J} / \psi$ nuclear modification factor as a function of collision centrality in $\mathrm{p}-\mathrm{Pb}$ collisions at $\sqrt{s_{\mathrm{NN}}}=8.16 \mathrm{TeV}$, at forward and backward rapidities, will also be presented. These measurements will be compared with Run-1 (2009 - 2013) results as well as theoretical calculations and will be interpreted in terms of cold nuclear matter effects.
\end{abstract}

\section{Introduction}

Measuring quarkonia in pp collisions may help to understand their production mechanisms and probe the parton distribution functions (PDF), in particular the gluon's PDF down to low $\mathrm{x}$. These measurements also provide a reference to $\mathrm{p}-\mathrm{Pb}$ and $\mathrm{Pb}-\mathrm{Pb}$ measurements which in turn are used to quantify the properties of the quark-gluon plasma. In $\mathrm{p}-\mathrm{Pb}$ these same measurements may probe cold nuclear matter effects such as modification of the PDFs in nuclei, saturation and Cronin enhancement $[1,2]$.

When we measure quarkonia we have contributions from decays from higher mass resonances ${ }^{1}$ and decays from $\mathrm{b}$ hadrons. The decay contributions are typically referred to as non prompt $\mathrm{J} / \psi$ and $\psi(2 \mathrm{~S})$. The ALICE quarkonium measurements presented in this document are inclusive which implies that no separation is made between prompt and non-prompt particles.

\section{Apparatus}

The ALICE detector is described in detail in $[3,4]$. The detector subsystems relevant to the present analysis are the muon spectrometer, the Silicon Pixel Detector (SPD) corresponding to the two innermost layers of the Inner Tracking System (ITS), the ZDC detectors, the V0 scintillator hodoscopes and the T0 Cherenkov detectors.

Charmonia are measured in the $\mu^{+} \mu^{-}$decay channel at forward rapidity and down to $p_{\mathrm{T}}=0$ using the ITS for vertex determination, the Muon Trigger (MTR) for triggering, the Muon Chambers

\footnotetext{
^e-mail: astrid.morreale@ cern.ch

${ }^{1}$ For the $\mathrm{J} / \psi$ these decay contributions are the $\psi(2 \mathrm{~S})$ and the $\chi_{c}$.
} 

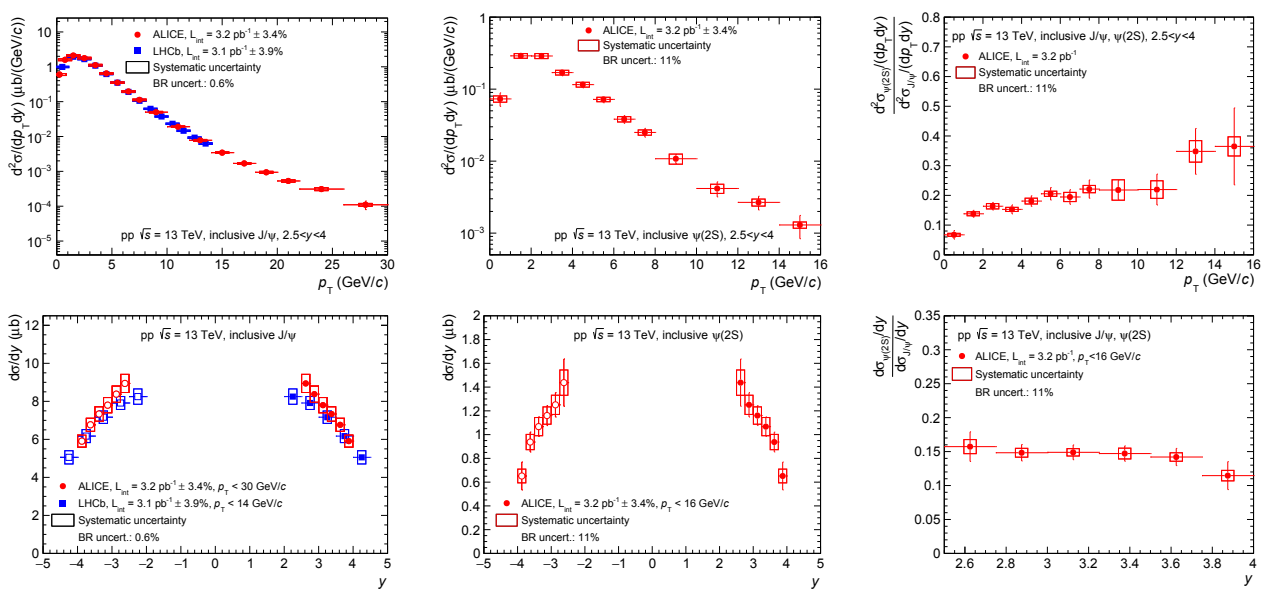

Figure 1. (color online). Inclusive $\mathrm{J} / \psi$ cross sections (left), $\psi(2 \mathrm{~S})$ cross sections (middle) and $\psi(2 \mathrm{~S})$-to- $\mathrm{J} / \psi$ cross section ratios (right) as a function of $p_{\mathrm{T}}$ (top panels) and $y$ (bottom panels) in pp collisions at $\sqrt{s}=13 \mathrm{TeV}$. $/ 4$ cross sections are compared to LHCb measurements at the same $\sqrt{s}$ [6]. Open symbols are the reflection of the positive- $y$ measurements with respect to $y=0$.

(MCH) for tracking. The V0 detectors are also used for triggering (in coincidence with the MTR), the T0 detectors are used for the luminosity determination and finally the V0 and ZDC detectors are used for the centrality determination in $\mathrm{p}-\mathrm{Pb}$ together with several charged particle multiplicity estimators defined in [5].

\section{$3 \mathrm{~J} / \psi$ and $\psi(2 \mathrm{~S})$ production in pp collisions}

Quarkonia are measured using fits to the invariant mass distribution of $\mu^{+} \mu^{-}$pairs detected in the muon system and at forward rapidity. The data sample analyzed for the measurements at $\sqrt{s}=13$ $\mathrm{TeV}$ correspond to an integrated luminosity $L_{\mathrm{int}}=3.19 \pm 0.11 \mathrm{pb}^{-1}$.

Figure 1 summarizes the inclusive $\mathrm{J} / \psi$ and $\psi(2 \mathrm{~S})$ cross sections as a function of the charmonium transverse momentum $p_{\mathrm{T}}$ (top figures) and rapidity $y$ (bottom figures). The left column shows the $\mathrm{J} / \psi$ cross sections while the middle and rightmost columns show the $\psi(2 \mathrm{~S})$ cross sections and the $\psi(2 \mathrm{~S})$-to- $\mathrm{J} / \psi$ cross section ratios respectively.

The $\mathrm{J} / \psi$ production cross sections as a function of $p_{\mathrm{T}}$ and $y$ are compared to measurements published by $\mathrm{LHCb}[6]$ at the same energy. The measurements of the two experiments are consistent within $1 \sigma$ of their uncertainties. The ALICE measurement extends the $p_{\mathrm{T}}$ reach from $14 \mathrm{GeV} / c$ to $30 \mathrm{GeV} / c$ with respect to the $\mathrm{LHCb}$ results. For the $\psi(2 \mathrm{~S})$ measurement, no comparisons are performed as this is the only measurement available to date at this energy and $y$ range. In Fig. 2, the cross sections presented in the previous section are compared to other forward- $y$ measurements in $\mathrm{pp}$ collisions at $\sqrt{s}=2.76$ [7], 5.02 [8], 7 [9] and $8 \mathrm{TeV}$ [10].

All ALICE $\mathrm{J} / \psi$ and $\psi(2 \mathrm{~S})$ measurements presented are inclusive and consist of a prompt and a non-prompt contribution. In order to compare model calculations to the data both contributions must be accounted for. This is illustrated in Fig. 3 for the $\mathrm{J} / \psi$ production cross section as a function of $p_{\mathrm{T}}$ in pp collisions at $\sqrt{s}=13 \mathrm{TeV}$.

In the left panel of Fig. 3, ALICE data at $\sqrt{s}=13 \mathrm{TeV}$ are compared to three calculations: i) in grey to a prompt $\mathrm{J} / \psi$ Next-to-Leading-Order (NLO) NRQCD calculation from Ma, Wang and Chao [11], ii) in blue to a prompt $J / \psi$ Leading Order (LO) NRQCD calculation coupled to a Color Glass Condensate (CGC) description of the low- $x$ gluons in the proton from Ma and Venugopalan [12] 

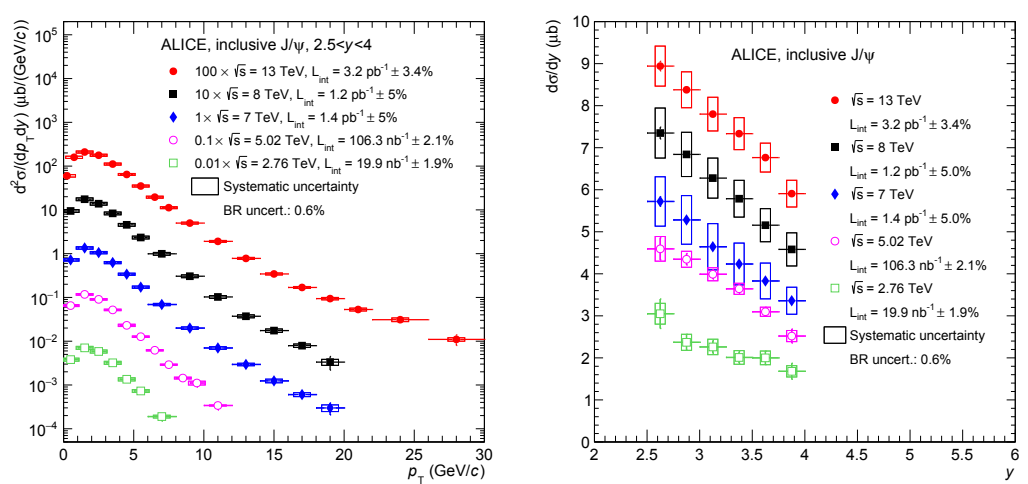

Figure 2. (color online). Inclusive $\mathrm{J} / \psi$ cross sections as function of $p_{\mathrm{T}}$ (left) and $y$ (right).
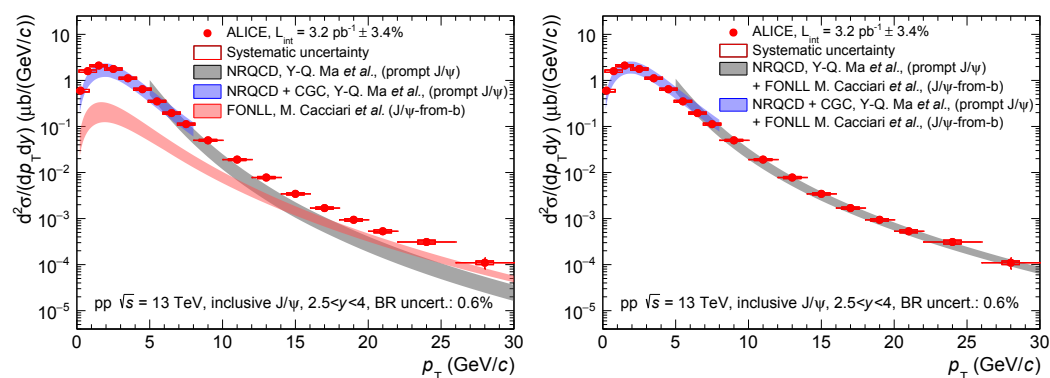

Figure 3. Left: ALICE data compared to NLO NRQCD (grey) [11], LO NRQCD coupled with CGC (blue) [12] and FONLL (red) [13]. Right: Summed NRQCD and FONLL predictions compared to the same data.

and iii) in red to a non-prompt $\mathrm{J} / \psi$ Fixed-Order Next-to-Leading Logarithm (FONLL) calculation by Cacciari et al. [13]. The summed NRQCD and FONLL calculations agree with ALICE data over the full $p_{\mathrm{T}}$ range and five orders of magnitude in $\sigma$.

\section{$4 \mathrm{~J} / \psi$ production in $\mathrm{p}-\mathrm{Pb}$ collisions at $\sqrt{s_{\mathrm{NN}}}=8.16 \mathrm{TeV}$}

In 2016, ALICE took data in two beam configurations corresponding to the proton momentum (p$\mathrm{Pb}$ collisions) or to the lead-nucleus momentum ( $\mathrm{Pb}-\mathrm{p}$ collisions) beam oriented towards the muon spectrometer. Measurements were obtained of the nuclear modification factor $R_{\mathrm{pPb}}$ (Fig. 4) of $\mathrm{J} / \psi$ at forward $\left(2.03<y_{\mathrm{cms}}<3.53\right)$ and backward $\left(-4.46<y_{\mathrm{cms}}<-2.96\right)$ rapidity corresponding to an $x$ region of $1.1 \times 10^{-5}<x<5 \times 10^{-5}(\mathrm{p}-\mathrm{Pb})$ and $7.3 \times 10^{-3}<x<3.3 \times 10^{-2}(\mathrm{~Pb}-\mathrm{p})$.

The corresponding data samples for the reported results corresponds to an integrated luminosity of $L_{\mathrm{int}} \mathrm{pPb}^{\mathrm{Pb}}=7.3 \mathrm{nb}^{-1}$ for $\mathrm{p}-\mathrm{Pb}$ collisions and $L_{\mathrm{int}} \mathrm{Pbp}=10.2 \mathrm{nb}^{-1}$ for $\mathrm{Pb}-\mathrm{p}$ collisions.

The results reported in Fig. 4 (top) extend the $p_{\mathrm{T}}$ range from 7 to $20 \mathrm{GeV} / c$ from the previous lower energy result at $\sqrt{s_{\mathrm{NN}}}=5.02 \mathrm{TeV}$ [14]. These new measurements are compared to theoretical calculations which include effects due to PDF modifications, color glass condensate, energy loss and final state effects. All models describe the data within the theoretical uncertainties [14].

The new preliminary results at $\sqrt{s_{\mathrm{NN}}}=8.16 \mathrm{TeV}$ confirm with an increased precision the features already observed at $\sqrt{s_{\mathrm{NN}}}=5.02 \mathrm{TeV}$ (Fig. 4 bottom). Mainly that the nuclear modification factor as function of centrality is smaller than one for forward rapidity and near unity for backward rapidity.

\section{Summary}

The inclusive $\mathrm{J} / \psi$ and $\psi(2 \mathrm{~S})$ differential cross sections as well as $\psi(2 \mathrm{~S})$-to-J $/ \psi$ cross section ratios as a function of $p_{\mathrm{T}}$ and $y$ measurements have been presented in pp collisions at $\sqrt{s}=13 \mathrm{TeV}$ with 

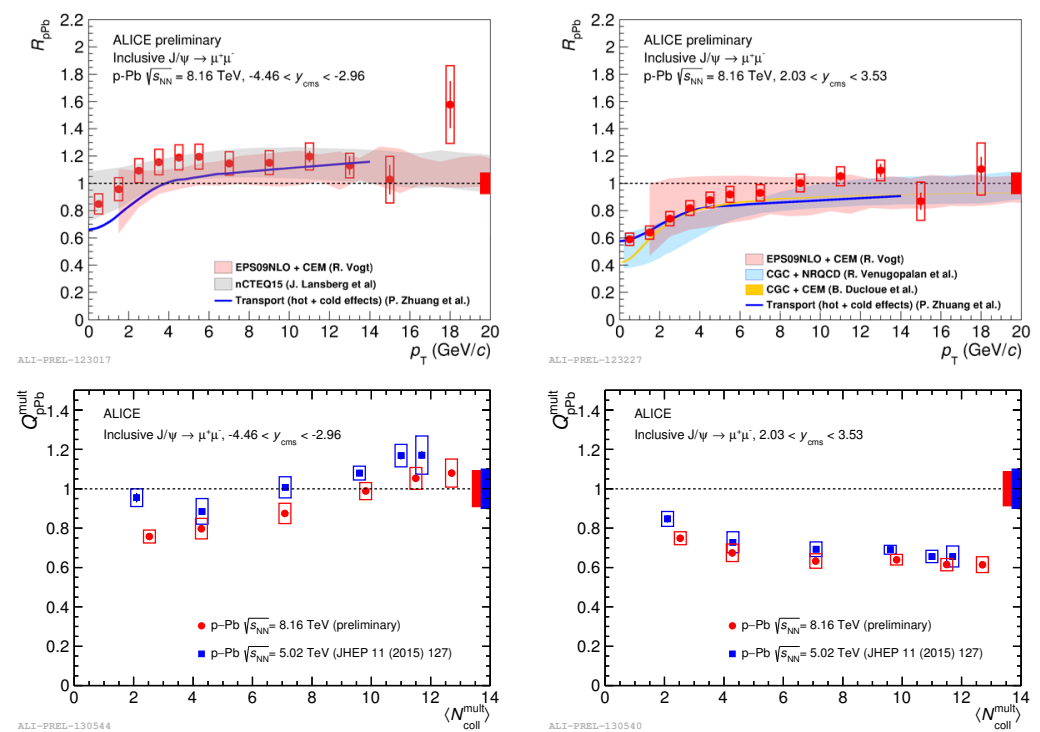

Figure 4. Top: $\mathrm{J} / \psi$ 's $R_{\mathrm{pPb}}$ as a function of $p_{\mathrm{T}}$ at backward (left) and forward rapidity (right). Bottom: same measurements as a function of centrality and compared between two center-of-mass energies $\sqrt{s_{\mathrm{NN}}}=8.16$ and $5.02 \mathrm{TeV}$. See text for details.

the ALICE detector. Comparisons of these measurements to NLO NRQCD and LO NRQCD+CGC prompt-charmonium calculations have been presented. Agreement is observed between data and theory for both particles and as a function of both $p_{\mathrm{T}}$ and $y$ provided that the non-prompt contribution to the inclusive cross section is included using FONLL. We have presented the measurement of the centrality dependence of inclusive $\mathrm{J} / \psi$ production in $\mathrm{p}-\mathrm{Pb}$ collisions at $\sqrt{s_{\mathrm{NN}}}=8.16 \mathrm{TeV}$. At forward rapidity, preliminary results show a suppression which slightly increases towards central collisions. At backward rapidity a different trend is observed, with the nuclear modification factor increasing from peripheral to central collisions. Results obtained at this new $\sqrt{s_{\mathrm{NN}}}$ are compatible with those previously measured by ALICE in $\mathrm{p}-\mathrm{Pb}$ collisions at $\sqrt{s_{\mathrm{NN}}}=5.02 \mathrm{TeV}$.

\section{References}

[1] N. Brambilla et al., Eur. Phys. J. C 71, 1534 (2011), 1010.5827

[2] A. Andronic et al., Eur. Phys. J. C76, 107 (2016), 1506.03981

[3] K. Aamodt et al. (ALICE Collaboration), JINST 3, S08002 (2008)

[4] B. Abelev et al. (ALICE Collaboration), Int. J. Mod. Phys. A29, 1430044 (2014), 1402 . 4476

[5] J. Adam et al. (ALICE Collaboration), Phys. Rev. C91, 064905 (2015)

[6] R. Aaij et al. (LHCb Collaboration), JHEP 10, 172 (2015), [Erratum: JHEP05,063(2017)], 1509.00771

[7] B. Abelev et al. (ALICE Collaboration), Phys. Lett. B718, 295 (2012), [Erratum: Phys. Lett.B748,472(2015)], 1203.3641

[8] J. Adam et al. (ALICE Collaboration), Phys. Lett. B766, 212 (2017), 1606.08197

[9] B.B. Abelev et al. (ALICE Collaboration), Eur. Phys. J. C74, 2974 (2014), 1403. 3648

[10] J. Adam et al. (ALICE Collaboration), Eur. Phys. J. C76, 184 (2016), 1509.08258

[11] Y.Q. Ma, K. Wang, K.T. Chao, Phys. Rev. Lett. 106, 042002 (2011), 1009. 3655

[12] Y.Q. Ma, R. Venugopalan, Phys. Rev. Lett. 113, 192301 (2014), 1408. 4075

[13] M. Cacciari et al., JHEP 10, 137 (2012), 1205.6344

[14] ALICE-PUBLIC-2017-007 (2017), https://cds.cern.ch/record/2272151 